\title{
Exact stability chart of an elastic beam subjected to delayed feedback
}

\author{
Li Zhang ${ }^{\mathrm{a}}$, Gabor Stepan ${ }^{\mathrm{b}, *}$ \\ a State Key Laboratory of Mechanics and Control of Mechanical Structures, Nanjing University of Aeronautics and Astronautics, \\ Nanjing 210016, People's Republic of China \\ ${ }^{\mathrm{b}}$ Department of Applied Mechanics, Budapest University of Technology and Economics, Budapest 1521, Hungary
}

\section{A R T I C L E I N F O}

\section{Article history:}

Received 31 August 2015

Received in revised form

1 December 2015

Accepted 4 January 2016

Handling Editor: D.J. Wagg

\begin{abstract}
A B S T R A C T
The stability of an elastic beam is studied when the beam is subjected to a longitudinal force governed by a feedback loop. The setup of the simplified mechanical model is motivated by a basic problem of electroacoustics. The corresponding governing equation is the 1D wave equation with delayed boundary conditions. By means of the D'Alembert solution, the system can be transformed into a delay differential equation of neutral type that includes two time delays. The intricate stability chart is constructed analytically in the parameter plane of the gain parameter and the ratio of the time delays. The complete chart extends the numerical results of the literature, while it also draws attention to the numerical difficulties of finite degree of freedom approximations and to the extreme sensitivity of the dynamics with respect to the time delay parameters of the system.
\end{abstract}

(c) 2016 Elsevier Ltd. All rights reserved.

\section{Introduction}

Dynamic properties of continua are often studied by means of finite element analysis and modal decomposition. These standard methods transform the original partial differential equation (PDE) into a large system of ordinary differential equations (ODE). However, as the size of the system increases, some properties of the finite degree of freedom (DoF) approximations may not tend to those of the continuum model. This is a critical issue often addressed in the literature [1-4].

In practical applications, feedback loops are frequently used to reduce vibrations in continuum dynamical systems, which is an especially hard task if the internal damping is small like in acoustic systems (see, for example, [5,6]). Delay is an essential element in any feedback loop. The presence of this unavoidable time delay in the controlled continuum system may lead to governing equations that consist of partial differential equations with delayed boundary conditions. There exist many representative examples in the literature. The study of Ma and Butcher [7] investigated the stability of a continuum beam when delayed follower force is applied at its end; in the paper of Haraguchi and Hu [8], the vibration suppression of a continuum beam is studied in the presence of delayed control. In both cases, they used low DoF approximations since the PDE models with delayed boundary conditions were too complex for analytical approaches. A basic microphone feedback system was also modelled as a chain of rigid blocks with elastic springs and time delay in the control loop in [9], which again leads to a finite DoF approximation of a wave equation with delayed boundary conditions. The results showed discrepancies in convergence as the DoF was increased.

\footnotetext{
* Corresponding author. Tel.: + 361463 1369; fax: + 3614633471 .

E-mail address: stepan@mm.bme.hu (G. Stepan).
} 
An alternative route in the analysis of these controlled continua is based on travelling wave solutions of the PDE part of the model. As representative mechanical examples, we mention the report in [10] that presents controllability conditions for a flexible beam in robotic applications, and the paper [11] of Szalai that studies the impact mechanics of elastic structures with delay models. An interesting experimental study has been published recently by Schmitz in [6] where the analogy of the squeal phenomenon in public address systems and the regenerative vibrations of machine tools was explored. The essential elements of the analogy are the existence of travelling waves and related time delays in both systems. Vibration suppression in both cases has a vast literature, here we refer only to spindle speed variation (the inverse of time delay variation) in machining processes [12,13], and to echo cancellation [14-16] or noise control [17-19] in electroacoustics.

The unavoidable occurrence of even a tiny time delay often complicates the desired stability properties of delay-free dynamical systems as analyzed in case of active vibration absorbers in [20,21]. Datko [22] showed it already in the 1980s that feedback stabilized hyperbolic systems were not robust with respect to small delays [23,24]. There are many intricate cases like these, which are explored by the methods developed for the stability analysis of linear neutral delay differential equations (NDDEs) (see the book [25] of Michiels and Niculescu or the series of publications by Olgac and Sipahi $[26,27]$ ). Since in case of NDDEs, the highest derivative of the state variable also appears in delayed form, infinitely many characteristic exponents can be located in the right half of the complex plane. This property makes these systems very sensitive for the variation of certain parameters, especially for the variation of the ratios of multiple time delays.

The rigorous mathematical result of Hale and Lunel [28] in 2002 showed that the linear difference equations with finite number of discrete delays are almost always unstable when the sum of the absolute values of the weights of the delayed terms is large enough, actually, if this sum is greater than 1 . These difference equations serve as the essential parts $[29,30]$ (also called associated delay-difference equations [25]) of the NDDEs, while the governing PDEs of continua can often be transformed to NDDEs by means of the travelling wave solutions [31,32]. Consequently, undamped continuum systems with delayed boundaries can be unstable for any irrational ratios of the arising time delays in the system. Still, a few zeromeasure (in engineering terms, zero-probability) stability domains were identified in some numerical examples, while these tiny stable domains could not be obtained through finite DoF approximations [9]. Similar numerical examples were presented also in section 1.2.5 of [25] related to the continuity properties of the spectrum with respect to the time delays.

The aim of this paper is to determine the complete and exact stability chart for the delayed boundary value problem modelling a controlled but otherwise undamped elastic beam. The chart is constructed in the parameter plane of the feedback delay and the feedback gain in closed mathematical form. This result also provides an explanation why these kinds of stability charts cannot be obtained by finite DoF approximations of the continuum beam. The physical and engineering relevance of the results are pointed out by means of the calculation of the closed form stability boundaries of the corresponding controlled beams in the presence of internal viscous damping.

The rest of the paper is organized as follows. In Section 2, the mechanical model of the controlled beam is presented including its geometry, material properties and the feedback parameters. The transformation of the equations of motion to NDDE is introduced in Section 3, where its essential part, a delay-difference equation with two discrete delays is also derived. In the subsequent sections, the preliminary results are summarized and the rigorous stability analysis is carried out for the undamped beam in case of rationally dependent time delays. The stability boundaries of the damped case are constructed analytically in Section 6, which leads to the concluding remarks on the numerical and engineering relevance of the results.

\section{Mechanical model}

The linearly elastic prismatic beam presented in Fig. 1 is subjected to a longitudinal load at its left end. The beam has length $l$, cross sectional area $A$; its material has density $\rho$ and modulus of elasticity $E$. The distributed state variable is denoted by $u(x, t)$ that describes the longitudinal displacement of a beam cross section at spatial position $x$ at time instant $t$. The normal force at the left end is governed by a feedback loop that amplifies the normal force sensed at the right end of the beam, which is otherwise fixed to a rigid wall. The feedback is characterized by the dimensionless gain $K$ and time delay $\tau$.

The governing equation of the longitudinal vibrations of the beam assumes the form

$$
\ddot{u}(x, t)-c^{2} u^{\prime \prime}(x, t)=0,
$$

where dot and prime denote derivatives with respect to the time $t$ and the space coordinate $x$, respectively. The speed of

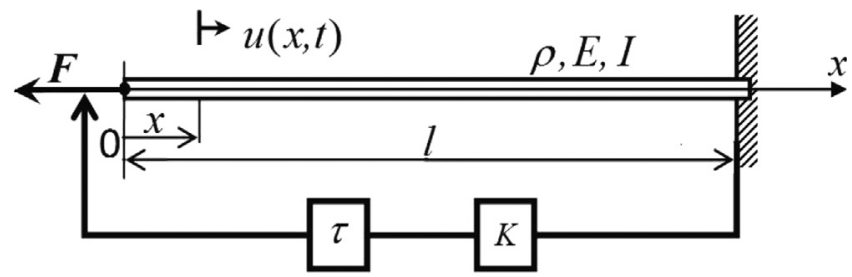

Fig. 1. Mechanical model of elastic beam subjected to delayed feedback. 
sound is given by

$$
c=\sqrt{E / \rho} .
$$

While the right end of the beam is fixed, i.e. $u(l, t)=0$, the vibrations are sensed there by means of the variation of the normal stress resultant $F(l, t)$ which is linearly proportional to the normal stress $\sigma(l, t)$ expressed by the measured strain $\epsilon(l, t)$ through Hooke's Law:

$$
F(l, t)=A \sigma(l, t), \quad \sigma(l, t)=E \epsilon(l, t), \quad \epsilon(l, t)=u^{\prime}(l, t) .
$$

This force is then fed back with gain $K$ and delay $\tau$ to the left end of the beam that results the normal stress resultant

$$
F(0, t)=K F(l, t-\tau)
$$

Consequently, the two boundary conditions of PDE (1) assume the form:

$$
\begin{gathered}
u(l, t)=0, \\
u^{\prime}(0, t)-K u^{\prime}(l, t-\tau)=0 .
\end{gathered}
$$

In case of public address systems mentioned in the Introduction, the microphone could be the sensor at the right end, while the loudspeaker may be the exciter at the left end. While the model is clearly over-simplified in this case, the consideration of linear characteristics at the boundaries and also at the amplifier between the sensor and the exciter is justified for linear stability analysis.

\section{D'Alembert solution}

The D'Alembert (or the so-called travelling wave) solution of Eq. (1) can be written in the form:

$$
u(x, t)=f(t-x / c)+g(t+x / c),
$$

where $f$ and $g$ are unknown scalar functions. The time needed for a wave of speed $c$ travelling along the beam is denoted by

$$
T=l / c \text {. }
$$

The substitution of the solution (7) into (5) and (6) results the new expressions of the boundary conditions:

$$
\begin{gathered}
f(t-T)+g(t+T)=0, \\
-\dot{f}(t)+\dot{g}(t)-K(-\dot{f}(t-\tau-T)+\dot{g}(t-\tau+T))=0 .
\end{gathered}
$$

Eq. (9) leads to a shift between the unknown functions

$$
g(t)=-f(t-2 T),
$$

with which one can transform Eq. (10) to an NDDE with respect to the unknown function $f$ in the form:

$$
\dot{f}(t)+\dot{f}(t-2 T)-2 K \dot{f}(t-T-\tau)=0 .
$$

The corresponding characteristic function $D(\lambda)$ with characteristic exponent $\lambda \in \mathbb{C}$ is obtained after the substitution of the exponential trial solution $f(t)=B e^{\lambda t}$ in (12):

$$
D(\lambda)=\lambda\left(1+\mathrm{e}^{-2 T \lambda}-2 K \mathrm{e}^{-(T+\tau) \lambda}\right) .
$$

Note that $\lambda=0$ corresponds to the trivial solution $u(x, t) \equiv 0$ independently from the corresponding constant values of the functions $f$ and $-g$ in Eqs. (11) and (7). By dropping this $\lambda=0$ trivial root, the characteristic function (13) results the characteristic equation

$$
1+\mathrm{e}^{-2 T \lambda}-2 K \mathrm{e}^{-(T+\tau) \lambda}=0 .
$$

This corresponds to a delay-difference equation with two time delays in it; one of the delays is $2 T$ that is the time needed for a wave to travel along the beam to and fro, while the other delay is $T+\tau$ where $\tau$ is the delay in the feedback loop. The continuous-time difference equation assumes the actual form

$$
y(t)+y(t-2 T)-2 K y(t-(T+\tau))=0,
$$

for the new variable $y=\dot{f}$. This is the essential part of the $\operatorname{NDDE}(12)$ (see $[25,29,30])$. In the present case, the condition $\operatorname{Re} \lambda<0$ for all the (infinitely many) characteristic exponents $\lambda$ satisfying the characteristic equation (14) is equivalent to the exponential stability of the essential part (15), which is equivalent to the exponential stability of the original PDE (1) with the delayed boundary conditions (5) and (6). 


\section{Preliminary results on stability}

In order to analyze the stability of the linear difference equation (15), we recall a basic theorem of Hale and Lunel [28]. For a scalar difference equation of the form

$$
x(t)-\sum_{k=1}^{m} a_{k} x\left(t-\tau_{k}\right)=0
$$

with rationally independent delays $\tau_{1}, \tau_{2}, \ldots, \tau_{m}$, the zero solution of Eq. (16) is exponentially stable if and only if

$$
\sum_{k=1}^{m}\left|a_{k}\right|<1
$$

Under this condition, the real parts of all the (infinitely many) characteristic roots $\lambda$ of the associated characteristic equation

$$
1-\sum_{k=1}^{m} a_{k} \mathrm{e}^{-\lambda \tau_{k}}=0
$$

are less than zero. The stabilizability of an NDDE having essential part of the form (16) and satisfying the condition (17) is studied in detail in [25-27]: if condition (17) does not fulfill, the corresponding linear NDDE cannot be exponentially stable for rationally independent delays.

In case of the characteristic equation (14) of the continuum beam subjected to delayed feedback, the corresponding time delays are

$$
\tau_{1}=2 T, \quad \tau_{2}=T+\tau .
$$

The sum of the absolute values of the coefficients of the delayed terms does not satisfy condition (17) since

$$
\sum_{i=1}^{2}\left|a_{i}\right|=|-1|+|2 K|=1+|2 K| \geq 1 .
$$

Consequently, if the time delays are rationally independent and the feedback gain $K$ is non-zero, the system governed by PDE (1) with boundary conditions (5) and (6) is exponentially unstable. However, when the time delays are rationally dependent, exponential stability is still possible as also shown in some numerical examples in [25] for specific cases like $\tau_{1}=1, \tau_{2}=2$. For the system governed by (15), numerical results in [9] show that $\tau$ values that are odd integer multiples of $T$ also result in exponential stability for certain gain parameters $K$.

As an attempt to confirm these numerical examples and to find all the exponentially stable domains in explicit form, the efforts will be put on the analytical stability investigation of the cases when $T$ and $\tau$ (and consequently, $\tau_{1}$ and $\tau_{2}$ in (19)) are rationally dependent. It is done in spite of the fact that these stability domains look physically irrelevant since they have zero-measure in the parameter plane, in other words, the slightest perturbation of the delay parameters leads to exponentially unstable systems. As already mentioned in Section 1, these domains are still important for checking the convergence and accuracy of numerical methods, and they will also serve as a basis for the study of systems that include internal damping.

\section{Stability analysis}

Consider the characteristic equation (14) with commensurable delay components $\tau$ and $T$. Then positive integers $p$ and $q$ can be found such that

$$
\tau / T=p / q, \quad p, q \in \mathbb{N}^{+} .
$$

Without loss of generality, we consider that $p$ and $q$ are coprimes. Then introduce the characteristic multiplier $\mu$ by

$$
\mathbb{C} \ni \mu:=\mathrm{e}^{h \lambda}, \quad \mathbb{R}^{+} \ni h:=\tau / p=T / q .
$$

This way, the stability condition expressed by means of the characteristic exponent $\lambda$ is transformed for the characteristic multiplier $\mu$ as

$$
\operatorname{Re} \lambda<0 \Leftrightarrow|\mu|<1,
$$

and the characteristic equation (14) assumes the form

$$
\mu^{p+q}+\mu^{p-q}-2 K=0 .
$$

Note that there is finite number $n=\max (p, q)+q$ of characteristic multipliers $\mu_{k}, k=1, \ldots, n$ that are obtained as roots of (24), while the number of the characteristic exponents

$$
\lambda_{k, j}=\frac{1}{h} \log \left|\mu_{k}\right|+\mathrm{i} \frac{1}{h}\left(\arctan \frac{\operatorname{Im} \mu_{k}}{\operatorname{Re} \mu_{k}} \pm 2 j \pi\right), \quad k=1, \ldots, n, j=1,2, \ldots
$$


is still infinite; they are uniformly distributed along finite number of vertical lines in the complex plane in accordance with (22).

In the special case of no feedback delay, that is when $\tau=0$, the corresponding characteristic equation can be simplified to

$$
\mu^{2 q}-2 K \mu^{q}+1=0 .
$$

It is known from Floquet Theory [25] that the system cannot be exponentially stable since the product of the characteristic multipliers must be 1 due to Vieta's 2nd formula; this case is also called marginal stability. Straightforward calculation shows that the condition of (marginal) stability is

$$
|\mu|=1 \Leftrightarrow-1 \leq K \leq 1 .
$$

In order to obtain an overview on the effect of the feedback delay $\tau>0$ on the stability of the system, the so-called stability chart is constructed in the plane of two parameters: the ratio $\tau / T(=p / q)$ and the gain $K$. The characteristic equation (24) is investigated in several steps. First, those critical values of the parameters are determined where some of the characteristic multipliers have absolute value $\left|\mu_{\mathrm{cr}}\right|=1$. Then the variation of the absolute value of each critical characteristic multiplier is determined for feedback gains close to their critical values $K_{\mathrm{cr}}$ based on the fact that the roots of polynomials depend continuously on the coefficients of the polynomials. This procedure is carried out first for critical gains at zero, then it is repeated for any large critical values of the gain $K$.

\subsection{Critical parameter values}

Consider the cases when critical characteristic multipliers $\left|\mu_{\mathrm{cr}}\right|=1$ exist. Then substitute

$$
\mu_{\text {cr }}=\mathrm{e}^{\mathrm{i} \alpha}
$$

with $\mathrm{i}^{2}=-1, \alpha \in \mathbb{R}$ into Eq. (24):

$$
\mathrm{e}^{\mathrm{i}(p+q) \alpha}+\mathrm{e}^{\mathrm{i}(p-q) \alpha}-2 K=0 .
$$

The corresponding real and imaginary parts satisfy the system of equations

$$
\begin{gathered}
\cos (p \alpha) \cos (q \alpha)-K=0, \\
\sin (p \alpha) \cos (q \alpha)=0 .
\end{gathered}
$$

From these, select the critical gain parameters $K_{\mathrm{cr}}$ in two cases:

$$
\begin{gathered}
\cos (q \alpha)=0 \Rightarrow \alpha_{j}=\frac{(2 j+1)}{2 q} \pi, \quad K_{\mathrm{cr}, *}=0, j=0 \ldots 2 q-1 \\
\sin (p \alpha)=0 \Rightarrow \alpha_{k}=\frac{k}{p} \pi, \quad K_{\mathrm{cr}, k}=(-1)^{k} \cos \left(\frac{k q}{p} \pi\right), \quad k=0 \ldots 2 p-1
\end{gathered}
$$

(a)

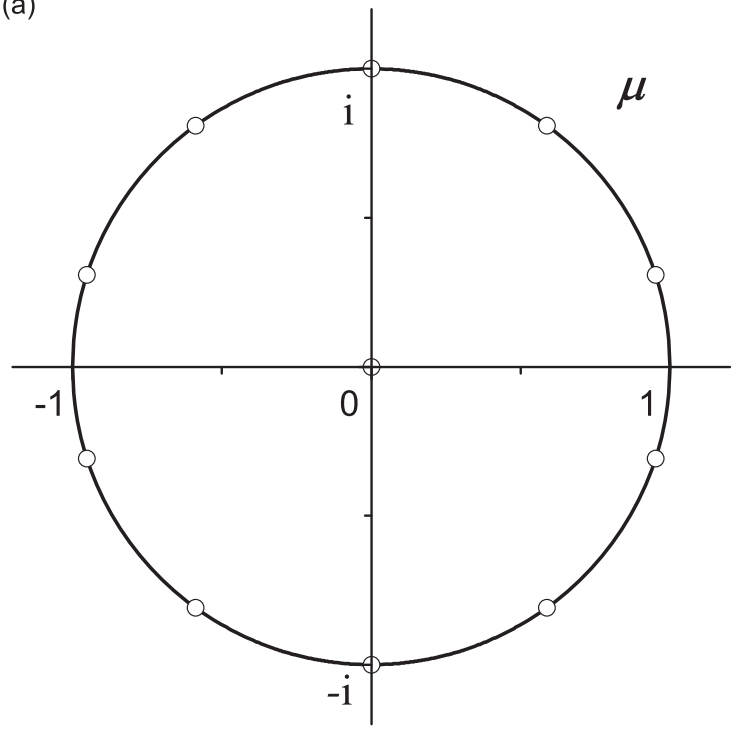

(b)

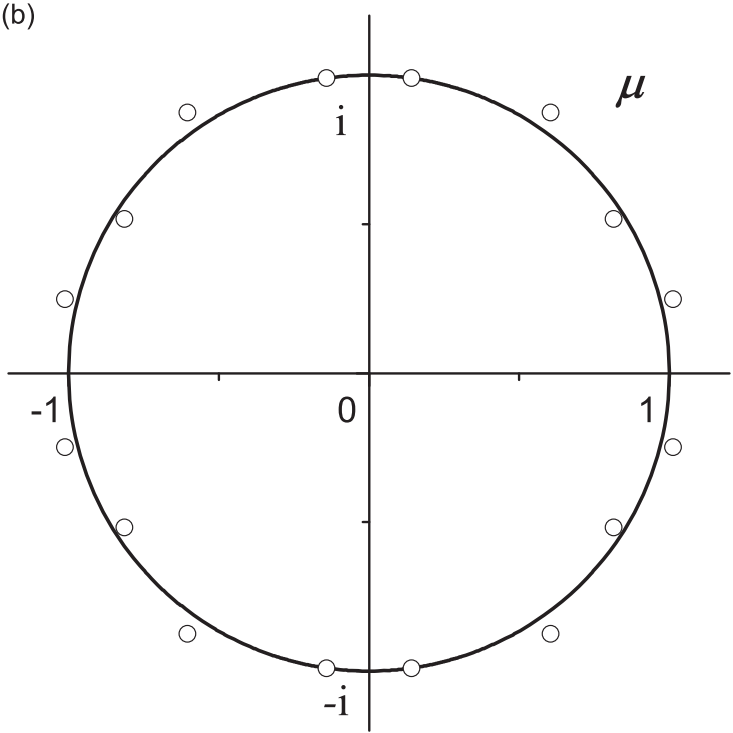

Fig. 2. Location of characteristic multipliers for $\tau / T=11 / 5$, (a) $K=K_{\mathrm{cr}, *}=0$ and (b) $K=K_{\mathrm{cr}, 17}=(-1)^{17} \cos (17 \cdot 5 \pi / 11)=\cos (8 \pi / 11)$. 
where $p$ and $q$ are optional positive (coprime) integers referring to any commensurable delay components $\tau$ and $T$ according to (21).

Typical arrangements of the characteristic multipliers in the complex plane are presented in Fig. 2. Panel (a) refers to a case when the critical gain is zero in accordance with formula (32). Note that there are further non-critical roots of (29); in case of the chosen parameters $p=11, q=5$, there are characteristic multipliers at zero with multiplicity $p-q=6$, apart from the $2 q=10$ critical roots on the unit circle given also in (32).

Panel (b) of Fig. 2 presents the characteristic multipliers for a non-zero critical gain $K_{\mathrm{cr}, k}$ in accordance with formula (33). Note that $\mu_{\text {cr }, k}=\mathrm{e}^{\mathrm{i} \alpha_{k}}$ in (33) is not the only critical root; in case of the chosen parameters $p=11, q=5, k=17$, one can find that $K_{\mathrm{cr}, 17}=K_{\mathrm{cr}, 5}=K_{\mathrm{cr}, 6}=K_{\mathrm{cr}, 16}$, which means that there are 4 critical roots $\mu_{\mathrm{cr}, 17,5,6,16}$ on the unit circle, apart from further 8 roots located outside and another 4 roots located inside. Although it is typical to have 4 critical roots at a critical gain $K_{\mathrm{cr}, k}$, it will be satisfactory to track only the corresponding $\mu_{\mathrm{cr}, k}$ given in (33).

At loss of stability, all the characteristic multipliers are located in the open unit disk except the critical ones that are located on the unit circle (see, for example, panel (a) of Fig. 2). Consequently, the boundaries of the stable parameter regions form a subset of all the possible parameters $p, q, K_{\mathrm{cr}}$ given in (32) and (33) where some of the characteristic multipliers are located on the unit circle independently whether the others are inside or outside (see, for example, panel (b) of Fig 2). In order to select the critical parameters at the stability boundaries only, the critical characteristic multipliers are checked whether they leave or enter or just graze the unit circle at the critical parameters as the gain $K$ is increased through its critical values (32) and (33). This property is also called root tendency in the literature [26].

\subsection{Crossing the unit circle at critical parameters}

The implicit differentiation of the characteristic equation (29) with respect to the gain parameter $K$ leads to

$$
\frac{\mathrm{d} \mu}{\mathrm{d} K}=\frac{2}{(p+q) \mu^{(p+q-1)}+(p-q) \mu^{(p-q-1)}} .
$$

The sense of crossing the unit circle of the complex plane, that is, the root tendency can be deduced from the variation of the absolute value of the characteristic multipliers:

$$
\frac{\mathrm{d}|\mu|}{\mathrm{d} K}=\frac{1}{|\mu|} \operatorname{Re}\left(\bar{\mu} \frac{\mathrm{d} \mu}{\mathrm{d} K}\right),
$$

where $\bar{\mu}$ denotes the complex conjugate of $\mu$. This derivative does not depend explicitly on the gain $K$ as shown by the substitution of (34) into (35). So when we check the sign of this derivative at the critical gain values $K_{\mathrm{cr}}$, the corresponding critical characteristic multipliers $\mu_{\mathrm{cr}}=\mathrm{e}^{\mathrm{i} \alpha}$ are substituted into (35) where the $\alpha$ values are given in (32) and (33). The algebraic manipulation results

$$
\left.\frac{\mathrm{d}|\mu|}{\mathrm{d} K}\right|_{\mu=\mathrm{e}^{\mathrm{i} \alpha}}=\frac{p \cos (p \alpha) \cos (q \alpha)-q \sin (p \alpha) \sin (q \alpha)}{p^{2}(1+\cos (2 q \alpha))+q^{2}(1-\cos (2 q \alpha))} .
$$

Clearly, if this derivative is positive (or negative) for a critical $\alpha$ given in (32) and (33), the corresponding characteristic multiplier $\mathrm{e}^{\mathrm{i} \alpha}$ crosses the unit circle outwards (or inwards, respectively).

If (36) is zero, the second derivative is needed, too. This can be calculated based on the formula

$$
\frac{\mathrm{d}^{2} \mu}{\mathrm{d} K^{2}}=-4 \frac{(p+q)(p+q-1) \mu^{p+q-2}+(p-q)(p-q-1) \mu^{p-q-2}}{\left((p+q) \mu^{p+q-1}+(p-q) \mu^{p-q-1}\right)^{3}}
$$

and by transforming it further to the absolute value of $\mu$ at $\mu_{\mathrm{cr}}$. The lengthy details of this calculation are not presented here.

The denominator of (36) is positive for any $\alpha$. At the critical parameters (32), the first term of the nominator is zero; in contrast, the second term is zero for (33). This way, the investigation of the root tendency number

$$
S:=\left.\operatorname{sign} \frac{\mathrm{d}|\mu|}{\mathrm{d} K}\right|_{\mu=\mathrm{e}^{\mathrm{i} \alpha}}
$$

is simplified separately for the case of (32) when the critical gain is always zero, and for (33) when several critical non-zero gain values exist.

\subsection{Critical gain at zero}

When $K=0$, the characteristic function (24) has critical roots of number $2 q$, which are uniformly distributed along the unit circle of the complex plane in accordance with formulae (28) and (32):

$$
\mu_{\mathrm{cr}, j}=\cos \left(\frac{2 j+1}{2 q} \pi\right)+\mathrm{i} \sin \left(\frac{2 j+1}{2 q} \pi\right), \quad j=0 \ldots 2 q-1
$$

(see also panel (a) of Fig. 2). Further roots exist for $p>q$, which are all at zero; there are no other roots when $K=0$. Thus, the 
system with zero gain is at the limit of stability, in other words, it is marginally stable for any positive integers $p$ and $q$; this is otherwise obvious for the original mechanical system without feedback, which is conservative.

With the help of the root tendency number $S$ of (36), those $p, q$ parameters can be identified where all the roots along the unit circle move inwards with increasing or decreasing gain $K$, that is, where regions of exponential stability exist. In accordance with the critical case (32), the calculation of (38) gives the root tendency number of the $j$ th critical root $\mu_{\mathrm{cr}, j}=\mathrm{e}^{\mathrm{i} \alpha_{j}}$ as

$$
S(j)=(-1)^{j+1} \operatorname{sign}\left(\sin \left(p \frac{2 j+1}{2 q} \pi\right)\right), \quad j=0 \ldots 2 q-1 .
$$

If $S(j)$ is +1 (or -1 ) for all $j$ then exponentially stable parameter domains exist as the gain $K$ decreases (or increases, respectively) from its zero value. If the sign of $S$ changes at certain $(j-1)$ st and $j$ th critical roots then some of the roots (39) move inwards the unit circle while others move outwards, consequently, there is no stable region there for small gain values $K$ around zero.

In summary, for given parameters $p$ and $q$,

(i) if $S(j)=+1$ for all values of $j$ then stable parameter domain exists for small negative gain values $K$;

(ii) if $S(j)=-1$ for all values of $j$ then stable parameter domain exists for small positive gain values $K$;

(iii) if there exists a value $j$ such that $S(j-1) S(j)=-1$ then there is no stable region there for small gain $K$;

(iv) if $S(j)=0$ for certain $j$ then the sign of the second derivative must be checked with the help of formula (37).

First, the case of odd $p$ is investigated while the coprime $q$ is optional, then the case of even $p$ is considered when the coprime $q$ can be an odd number only.

\subsubsection{Case of odd $p$}

If $q=1$, then (40) can be calculated as

$$
S(j)=(-1)^{j+1} \operatorname{sign}\left(\sin \left(p \frac{2 j+1}{2} \pi\right)\right)=(-1)^{(p+1) / 2},
$$

which is independent of the index $j$ of the roots, that is, all the critical roots move in the same direction from the unit circle. More specifically, $S=-1$ and all the critical roots move inwards for $p=1,5,9, \ldots$ presenting exponentially stable domains at small positive gains $K>0$ for $\tau / T=1,5,9, \ldots$ (see panel (a) of Fig. 3), while $S=+1$ and all the critical roots move outwards for $p=3,7,11, \ldots$ presenting exponentially stable domains at small negative gains $K<0$ for $\tau / T=3,7,11, \ldots$ (see panel (b) of Fig. 3). The corresponding domains can be identified as parts of the thick straight lines representing the exponentially stable parameter domains in the stability chart of Fig. 6 explained in detail later.

If $q \neq 1$ then there always exists $j$ such that $S(j-1) S(j)=-1$, which means that there always exist characteristic multipliers outside the unit circle for any small non-zero gain $K$ (see panel (a) of Fig. 4). The proof of this is presented in Appendix A.

(a)

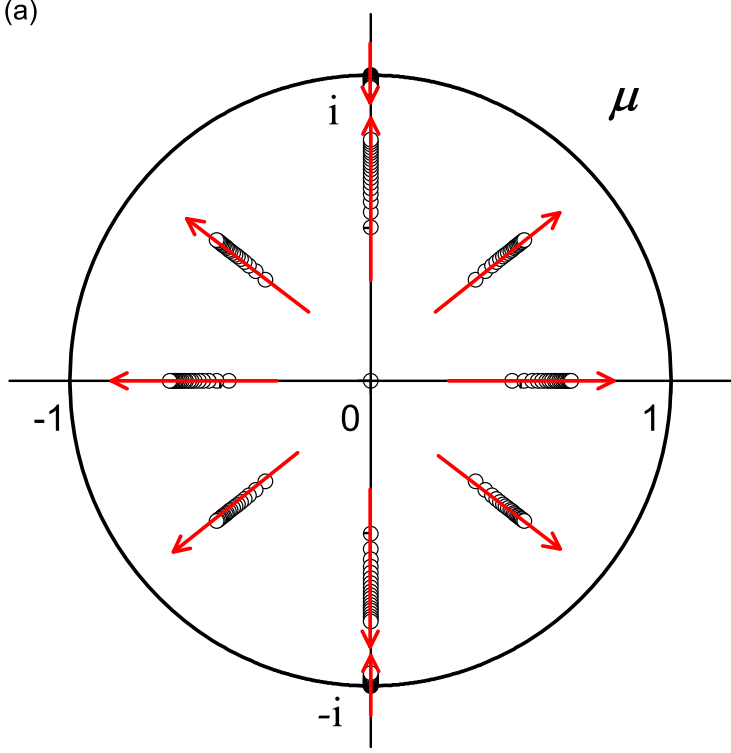

(b)

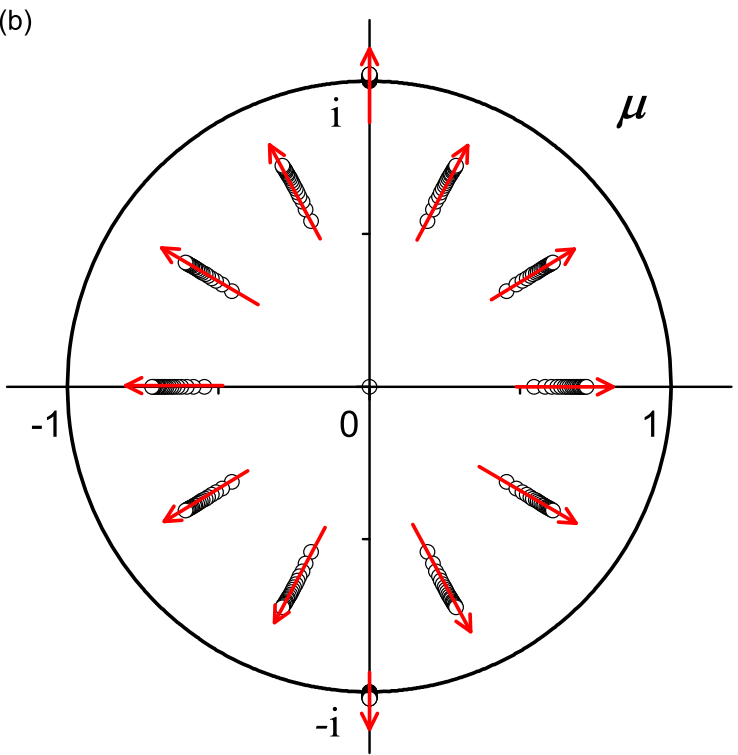

Fig. 3. Locus curves of all the characteristic multipliers as gain $K$ increases through $K_{\mathrm{cr}, *}=0$ for (a) $\tau / T=9$ when the critical roots at e \pm i $\pi / 2$ move inwards and for (b) $\tau / T=11$ when these critical roots move outwards the unit circle. 
(a)

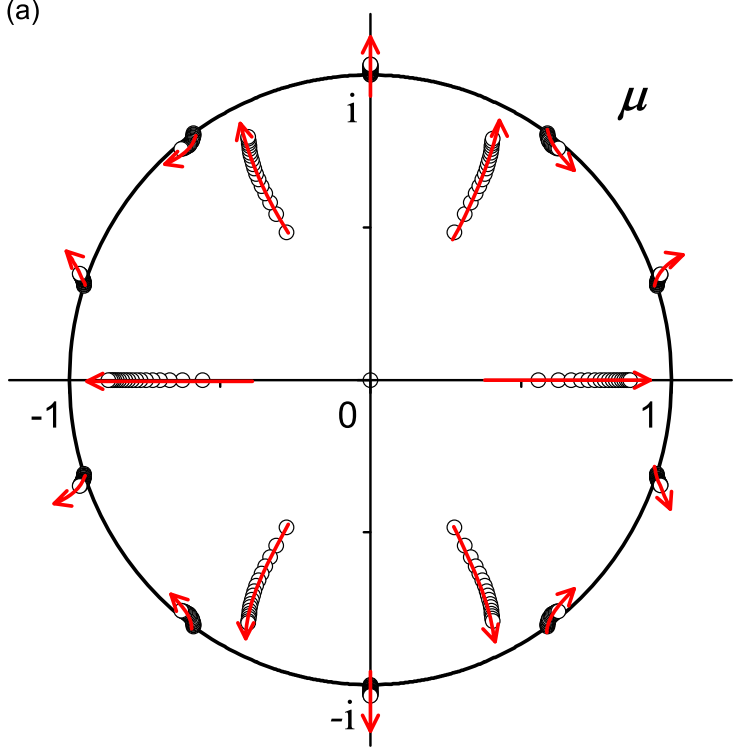

(b)

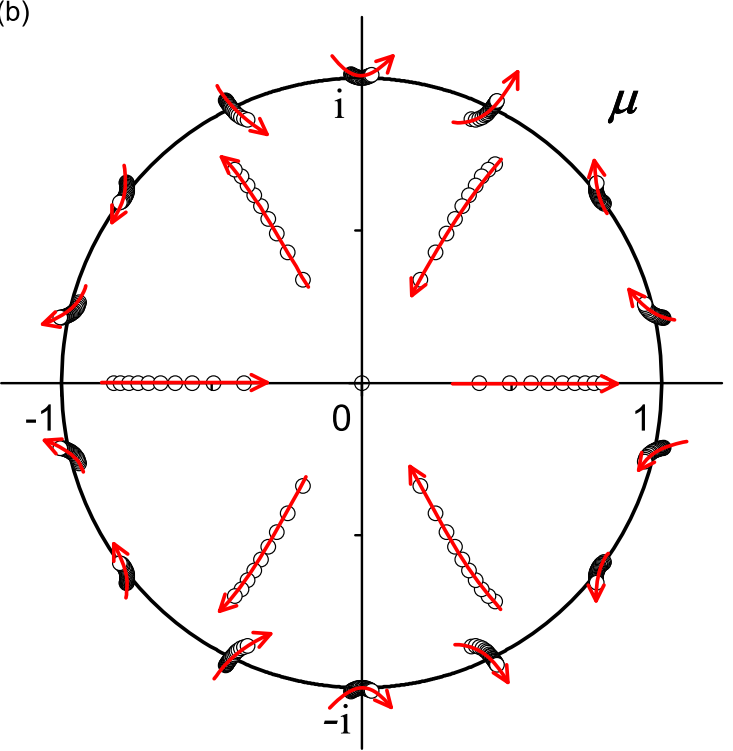

Fig. 4. Locus curves of characteristic multipliers as gain $K$ increases through $K_{\mathrm{cr}, *}=0$ for (a) $\tau / T=13 / 5$ when neighboring critical roots exist on the unit circle with opposite root tendencies, and for (b) $\tau / T=10 / 7$ when critical roots at $\mathrm{e}^{ \pm \mathrm{i} \pi / 2}$ graze the unit circle from outside.

(a)

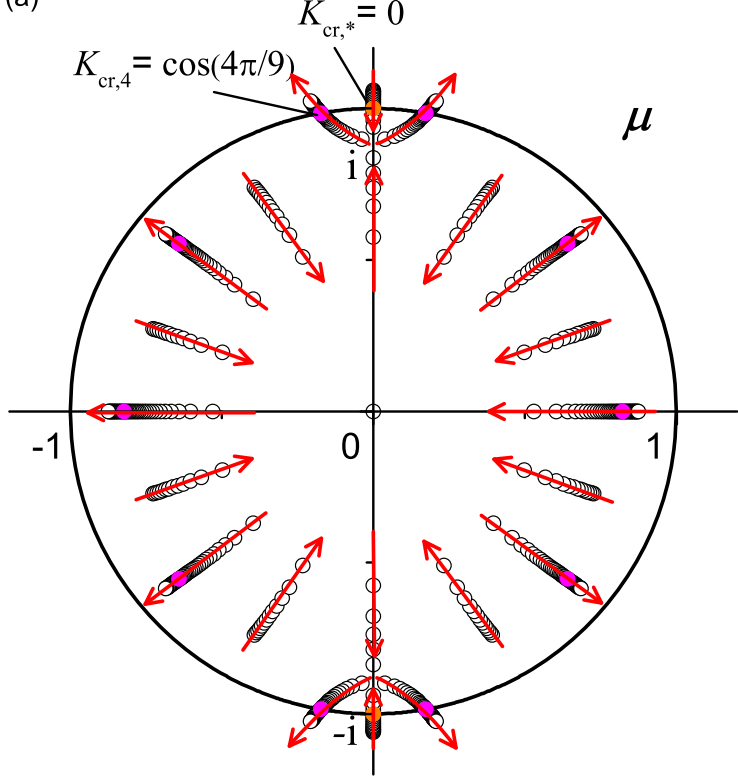

(b)

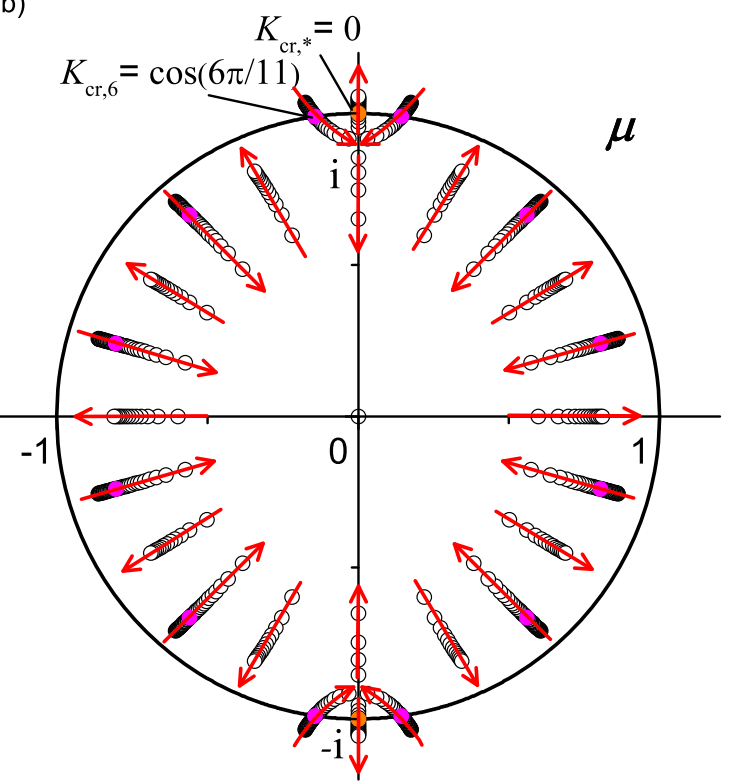

Fig. 5. Locus curves of characteristic multipliers for (a) $\tau / T=9, K=-0.1, \ldots, 0.3$ and for (b) $\tau / T=11, K=-0.3, \ldots, 0.1$.

\subsubsection{Case of even $p$}

If $p$ is even then the coprime $q$ must be odd. Consequently, there exists an integer $j=(q-1) / 2$ for which formula (32) of the critical cases presents a critical root with argument $\alpha_{j}=\pi / 2$. For this root

$$
\left.\frac{\mathrm{d}|\mu|}{\mathrm{d} K}\right|_{\mu=\mathrm{e}^{\mathrm{i} \pi / 2}}=0,
$$

since both $\cos (q \pi / 2)=0$ and $\sin (p \pi / 2)=0$ due to the odd $q$ and even $p$ substituted in (36). 
Thus, the second derivative of the modulus of $|\mu|$ with respect to $K$ is needed. This lengthy calculation can be carried out with the help of formula (37) that leads to the simple result

$$
\left.\frac{\mathrm{d}^{2}|\mu|}{\mathrm{d} K^{2}}\right|_{\mu=\mathrm{e}^{\mathrm{i}} / 2}=4 \frac{p}{q^{2}}>0
$$

This means that there is a characteristic multiplier that just grazes the unit circle from outside (see also panel (b) of Fig. 4), and consequently, all these cases are exponentially unstable for small non-zero gains $K$.

\subsection{Non-zero critical gains}

There is a set of critical parameters calculated in (33) where the critical gain values $K_{\mathrm{cr}, k}=(-1)^{k} \cos (q k \pi / p)$ are non-zero and the arguments of the critical roots are $\alpha_{k}=k \pi / p$ for all $k=0, \ldots, 2 p-1$. At these critical parameters, the root tendency number $S$ in (38) can easily be calculated from (36):

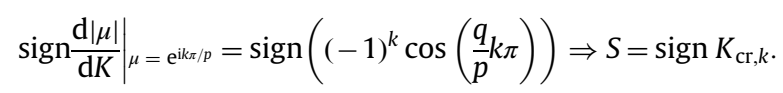

This means that the critical characteristic multipliers move outwards both for increasing positive gain values and for decreasing negative gains. Thus, the system cannot be stabilized by increasing absolute values of the gain, and the system loses stability at the first non-zero gain in those parameter domains where it is exponentially stable for near-zero gains. For positive gains, these critical stability boundaries are

$$
\min _{k=0, \ldots, 2 p-1} K_{\mathrm{cr}, k}=\cos \left(\frac{p-1}{2 p} \pi\right)>0, \quad p=1,5,9, \ldots,
$$

while for negative gains, the corresponding critical stability boundaries are

$$
\max _{k=0, \ldots, 2 p-1} K_{\mathrm{cr}, k}=\cos \left(\frac{p+1}{2 p} \pi\right)<0, \quad p=3,7,11, \ldots
$$

Corresponding representative examples are given in panels (a) and (b) of Fig. 5.

\subsection{Stability chart}

In the space of the system parameters, a stability chart presents those parameter domains where the actual equilibrium or motion of a system is stable. In case of the elastic beam subjected to delayed feedback at its boundaries, the system is governed by the linear PDE (1) with boundary conditions (5) and (6), and the exponential stability of the equilibrium can be represented in the parameter plane of the delay ratio $\tau / T$ and the feedback gain $K$. Based on the existing instability results of the literature for rationally independent delays, and the results of the above analyses of all the possible combinations of the rationally dependent (commensurable) delays, we can state that the only parameters where the equilibrium is exponentially

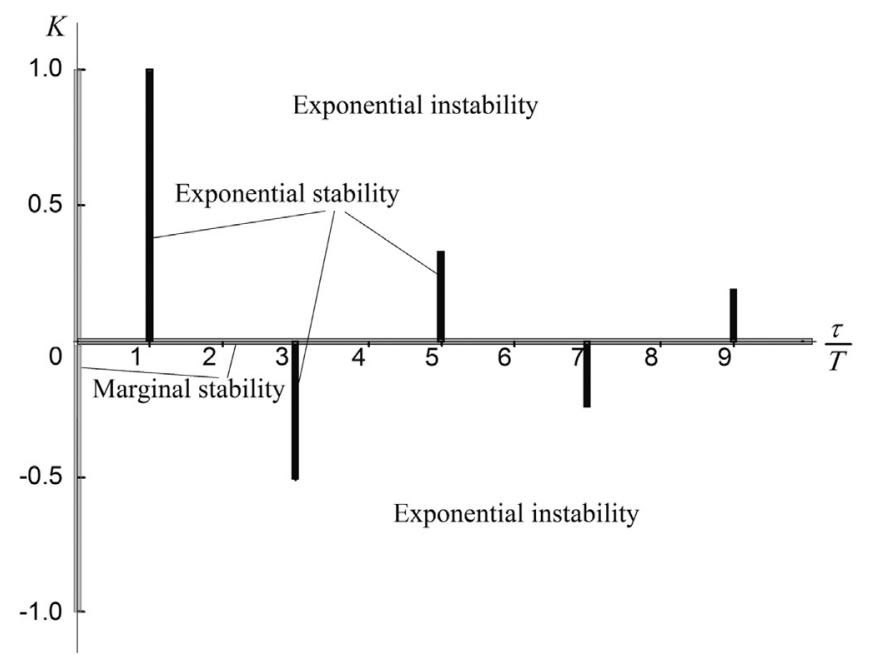

Fig. 6. Stability chart in the plane of the delay components ratio $\tau / T$ and the gain $K$. White regions refer to exponentially unstable domains, thick black lines represent exponentially asymptotically stable domains, and gray thick line at $\tau=0$ represents marginally stable domain. 
asymptotically stable are the following zero-measure sets:

$$
\tau / T=4 n-3, \quad 0<K<\cos \left(\frac{2 n-2}{4 n-3} \pi\right) \quad(n=1,2, \ldots)
$$

and

$$
\tau / T=4 n-1, \quad \cos \left(\frac{2 n}{4 n-1} \pi\right)<K<0 \quad(n=1,2, \ldots)
$$

where the non-zero bounds of the gain $K$ are obtained from the $\mathrm{min} / \mathrm{max}$ formulas in (45) and (46). These domains are presented in Fig. 6 together with the marginally stable regions $-1 \leq K \leq+1$ at $\tau=0$ (see (27)), and $\tau>0$ at $K=0$ where the uncontrolled system is conservative.

The stability chart follows the peculiar property predicted by the discontinuity of the spectrum with respect to the delays [25], that is, optionally small variation of the delay may cause the abrupt change of the system from exponentially asymptotically stable to exponentially unstable. In the meantime, the continuity of the spectrum is preserved with respect to the gain parameter; this is the reason why the stable domains have finite 'lengths' along the vertical axis of the chart. Although these stable regions are irrelevant from engineering view-point, the presence of some damping in the controlled beam may change them qualitatively. This is studied in the subsequent section, again, with closed form analytical results.

\section{Stability in the presence of damping}

There are several options to model damping effects in continua [33]. The most relevant ones are the so-called external damping when the dissipative forces are proportional to the velocities of the particles that are in contact with the environment, and the internal damping when the dissipative forces are proportional to the relative velocities between neighboring particles. Similarly, these damping forces could be of Coulomb-type. The case of viscous internal damping is considered here with a factor $\eta>0$ that describes the damping forces proportional to the internal elastic forces, while the absorption effects at the ends of the beam are neglected. This leads to the simplest possible mathematical model of the damped continuum beam in the form:

$$
\ddot{u}(x, t)-\eta c^{2} \dot{u}^{\prime \prime}(x, t)-c^{2} u^{\prime \prime}(x, t)=0
$$

with the same boundary conditions (5) and (6) as in the undamped case. The difficulty of the stability analysis of PDE (49) arises from the fact that the corresponding characteristic exponents are not located on finite number of vertical lines in the complex plane in contrast to the undamped case (see (25)), although their real parts still converge to finite number of values as their modulus tends to infinity (see [29]). Still, the stability boundaries can be found in explicit form.

Substitute the exponential trial solution $u(x, t)=U(x) \mathrm{e}^{\lambda t}$ into Eq. (49). Then the vibration mode $U(x)$ satisfies the ODE

$$
U^{\prime \prime}(x)-\frac{\lambda^{2}}{c^{2}(1+\eta \lambda)} U(x)=0
$$

with boundary conditions

$$
U(l)=0, \quad U^{\prime}(0)-K U^{\prime}(l) \mathrm{e}^{-\lambda \tau}=0
$$

inherited from (5) and (6). The corresponding general solution

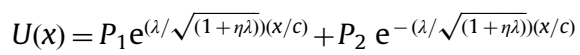

satisfies the boundary conditions (51) with non-trivial coefficients $P_{1,2}$ if

$$
\left[\begin{array}{cc}
\mathrm{e}^{(1 / \sqrt{(1+\eta \lambda)}) \lambda T} & \mathrm{e}^{-(1 / \sqrt{(1+\eta \lambda))}) \lambda T} \\
\lambda\left(1-K \mathrm{e}^{(1 / \sqrt{(1+\eta \lambda))}) \lambda T-\lambda \tau}\right) & -\lambda\left(1-K \mathrm{e}^{-(1 / \sqrt{(1+\eta \lambda))}) \lambda T-\lambda \tau}\right)
\end{array}\right]\left[\begin{array}{l}
P_{1} \\
P_{2}
\end{array}\right]=\left[\begin{array}{l}
0 \\
0
\end{array}\right] .
$$

The determinant of the leading matrix leads to the characteristic function

$$
D(\lambda)=\lambda\left(\cosh \frac{T \lambda}{\sqrt{1+\eta \lambda}}-K \mathrm{e}^{-\tau \lambda}\right)
$$

In case of the uncontrolled beam, $K=0$ and the characteristic equation simplifies to

$$
\mathrm{e}^{T \lambda / \sqrt{(1+\eta \lambda)}}+\mathrm{e}^{-T \lambda / \sqrt{(1+\eta \lambda)}}=0 \Rightarrow \frac{2 T \lambda}{\sqrt{1+\eta \lambda}}= \pm \mathrm{i}(2 k+1) \pi, \quad k=0,1, \ldots .
$$

Since the right-hand side involves both positive and negative values, the square of this equation leads to

$$
4 T^{2} \lambda^{2}+\eta(2 k+1)^{2} \pi^{2} \lambda+(2 k+1)^{2} \pi^{2}=0, \quad k=0,1, \ldots,
$$

from which the infinitely many characteristic exponents of the proportionally damped and uncontrolled beam can be calculated in closed form. Clearly, each value of $k$ represents a vibration mode, and each mode is associated with a viscous 


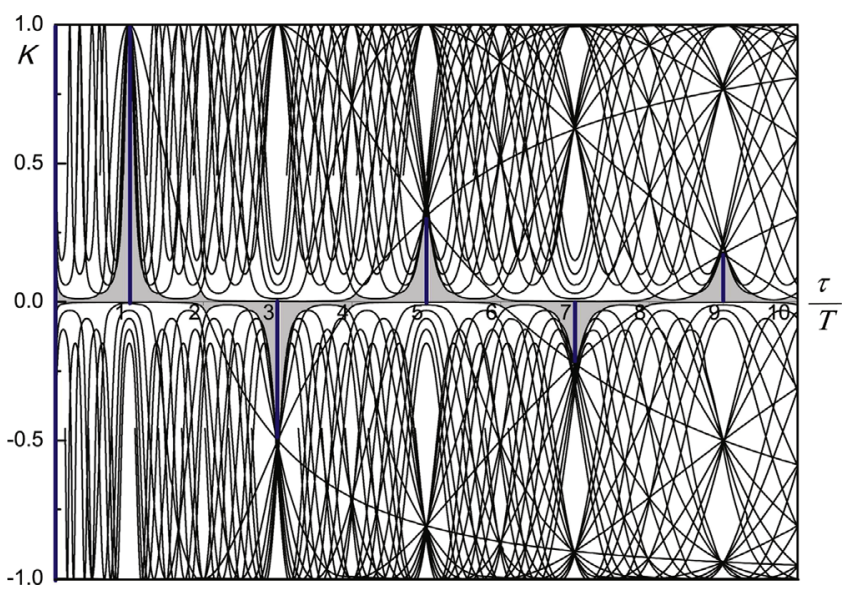

Fig. 7. The critical curves in the parameter plane of delay ratio $\tau / T$ and gain $K$ represent parameters where pure imaginary characteristic exponents exist for the dimensionless damping factor $\tilde{\eta}=0.001$. The regions where the trivial solution of (49) is exponentially stable are shaded in gray. Within these regions, the blue vertical lines represent the zero-measure stability domains for the undamped case $\tilde{\eta}=0$ as given in Fig. 6 . (For interpretation of the references to color in this figure caption, the reader is referred to the web version of this paper.)

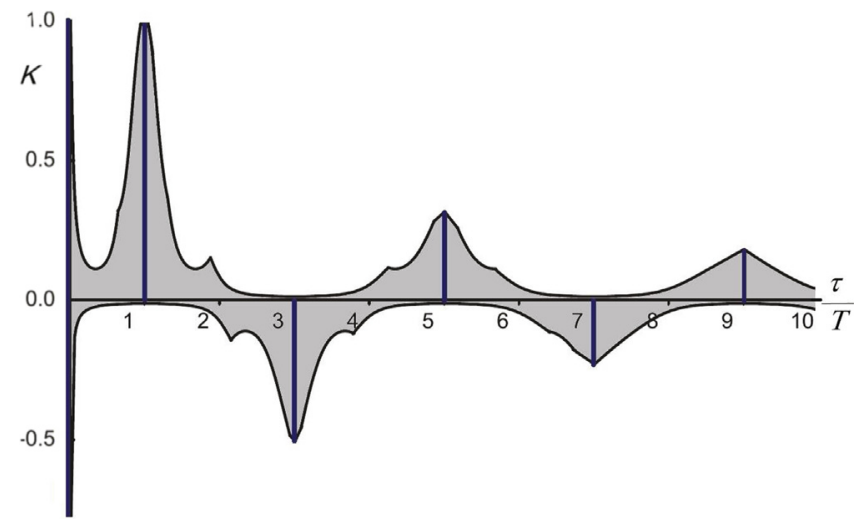

Fig. 8. Stability chart for dimensionless damping factor $\tilde{\eta}=0.01$. Gray regions refer to exponential stability; $K=0$ axis is also exponentially stable in the presence of damping. Vertical thick black lines refer to exponential stability in the undamped case.

damping proportional to the square of the corresponding natural frequency in accordance with the proportional nature of damping considered in the model. The Routh-Hurwitz criterion guarantees that Re $\lambda_{k}<0$ for all $k$ due to the positive coefficients of the polynomial in (56), which is in accordance with the exponential stability of the uncontrolled damped beam. This calculation cannot be repeated for $K>0$.

For the controlled $(K \neq 0)$ but undamped $(\eta=0)$ case, the characteristic function (54) simplifies to (13). Still, the analysis of (54) is much more difficult: the characteristic multiplier $\mu$ cannot be introduced since the infinitely many characteristic exponents are not arranged along vertical lines in the presence of damping.

Due to boundary condition (5), the characteristic exponent $\lambda=0$ of (54) corresponds to the trivial solution $u(x, t) \equiv 0$ only. Then the critical parameter domains where further pure imaginary characteristic exponents occur can be obtained by substituting $\lambda_{\mathrm{cr}}=\mathrm{i} \omega, \omega \geq 0$ in (54). The separation of the real and imaginary parts of $D(\mathrm{i} \omega)$ presents two equations similar to (30) and (31), but in a more complicated form. Still, the parameters $\tau$ and $K$ can be expressed explicitly:

$$
\begin{aligned}
& K_{\mathrm{cr}}^{2}=\sinh ^{2}\left(\frac{\omega T}{\sqrt{2}} \frac{\sqrt{\sqrt{\eta^{2} \omega^{2}+1}-1}}{\sqrt{\eta^{2} \omega^{2}+1}}\right)+\cos ^{2}\left(\frac{\omega T}{\sqrt{2}} \frac{\sqrt{\sqrt{\eta^{2} \omega^{2}+1}+1}}{\sqrt{\eta^{2} \omega^{2}+1}}\right), \\
& \tan \left(\omega \tau_{\mathrm{cr}}\right)=-\tanh \left(\frac{\omega T}{\sqrt{2}} \frac{\sqrt{\sqrt{\eta^{2} \omega^{2}+1}-1}}{\sqrt{\eta^{2} \omega^{2}+1}}\right) \tan \left(\frac{\omega T}{\sqrt{2}} \frac{\sqrt{\sqrt{\eta^{2} \omega^{2}+1}+1}}{\sqrt{\eta^{2} \omega^{2}+1}}\right) .
\end{aligned}
$$

These expressions are used to construct the stability chart of the damped controlled beam in the plane of the delay components ratio $\tau / t$ and the gain $K$ : the dimensionless frequency parameter $\tilde{\omega}=\omega T$ is swept in $(0, \infty)$ for selected (small) 
values of the dimensionless damping coefficient $\tilde{\eta}=\eta / T$ in (57) and (58) with the careful selection of the two-parameter arc tangent values and the corresponding signs of $K_{\mathrm{cr}}$. The critical parameter values are located on tangled curves in the parameter plane as presented in Fig. 7 for dimensionless damping factor $\tilde{\eta}=0.001$.

The diagram also presents the stable regions (lines) for the undamped case, which fit perfectly among these curves.

Clearly, a correct mathematical proof for the exact stability chart of the damped controlled beam requires further extensive algebraic work that can be carried out by computer algebra only following the procedure developed for the undamped case: along each curve, the root tendency has to be calculated. The critical characteristic exponents all move to the right of the complex plane either for increasing positive gains or for decreasing negative gains. The stable regions are shaded in gray in Fig. 7 accordingly. Alternatively, the continuity of the spectrum has to be proven for the damped beam in the presence of delayed feedback, which is not true for the undamped case.

For a further increased dimensionless damping ratio $\tilde{\eta}=0.01$, the stability chart is presented in Fig. 8 where the gray regions refer to exponentially asymptotically stable systems, even for the uncontrolled case of $K=0$. Note that apart from the widening regions of stability around the stability lines of the undamped system, there are 'peaks' of enlarged stable domains around the even delay ratios $\tau / T=2,4, \ldots$ where the undamped system gets close to being marginally stable with characteristic multipliers grazing the unit circle from outside.

\section{Concluding remarks}

It is known from recent mathematical results of the literature that

- linear neutral delay systems with rationally independent delays are always unstable if the sum of the moduli of the corresponding coefficients is larger than 1 [28];

- the spectrum of these systems is not continuous with respect to the delay parameters [25];

- there exist commensurable delays where the above systems are exponentially asymptotically stable even if the sum of the moduli of the corresponding coefficients is larger than $1[9,25]$.

\section{We have shown that}

(i) there are realistic physical models that lead to the above described critical mathematical cases, like the governing equations of the elastic beam subjected to delayed feedback at its ends;

(ii) the exact stability chart constructed from explicit calculations in the plane of the control gain and the delay ratio consists of discrete, uniformly spaced zero-measure lines at odd delay ratios where the system is exponentially stable while the slightest perturbation of the delay parameters result exponentially unstable systems;

(iii) these physically irrelevant stable domains become relevant as they open up to finite regions of exponential stability in the presence of internal damping, while the stability boundaries form fractal-like structures.

The stability of the wave equation with delayed boundary condition serves as a paradigm for a class of dynamical stability problems often leading to intricate self-excited vibrations. While the squeal phenomenon in public address systems is an obvious element of this class, many advanced models of elastic structures under retarded follower forces [34,7], directional drilling tasks $[35,36]$ and machine tool chatter suppression $[37,38]$ belong there, too. The results and the applied methodology could also serve as a basis for system parameter identification of continuum beams and shells by means of self-excited vibrations induced with the help of delayed feedback loops.

The dynamical properties of these systems are still unexplored in spite of the large computational efforts based on finite DoF approximations like finite element analysis and experimental modal analysis. Our closed form analytical results also explain why the finite DoF approximations do not converge, or why they do converge very slowly during the numerical treatment of these problems. This way, our results also provide an effective numerical challenge for testing the limits of current dynamics software.

\section{Acknowledgments}

This research was supported partially by the European Research Council under the European Union's Seventh Framework Programme (FP/2007-2013) ERC Advanced Grant agreement no. 340889, and the Hungarian-Chinese Bilateral Scientific and Technological Cooperation Fund under Grant no. TET_12_CN-1-2012-0012. The work of L.Z. was supported by the National Natural Science Foundation of China under Grant nos. 11302098 and 11172126, the work of G.S. was also supported by the Hungarian Scientific Research Foundation OTKA under Grant no. K101714.

The authors acknowledge with thanks the discussions with Prof. Janos Turi on the semigroup approximations of partial and functional differential equations. 


\section{Appendix A. Proof of instabilities in Section 5.3.1}

When $p$ is odd and the coprime $q \neq 1$ then calculate the product of the signs by means of formula (41):

$$
S(j-1) S(j)=-\operatorname{sign}\left(\sin \left(\frac{2 j-1}{2} \frac{p}{q} \pi\right) \sin \left(\frac{2 j+1}{2} \frac{p}{q} \pi\right)\right)=-\operatorname{sign}\left(\frac{1}{2}\left(\cos \left(\frac{p}{q} \pi\right)-\cos \left(2 j \frac{p}{q} \pi\right)\right)\right)
$$

We show in four steps that there always exist a certain $j$ such that the above product is -1 , that is, there exists $j$ such that

$$
\cos \left(2 j \frac{p}{q} \pi\right)<\cos \left(\frac{p}{q} \pi\right)
$$

(i) If $(0<) p<q$ then choose $j$ according to

$$
\frac{p}{2(q-p)}<j<\frac{2 q-p}{2(q-p)} \Leftrightarrow 2 j \pi-\frac{p}{q} \pi>2 j \frac{p}{q} \pi>(2 j-1) \pi+\frac{p}{q} \pi \Leftrightarrow \cos \left(2 j \frac{p}{q} \pi\right)<\cos \left(2 j \pi-\frac{p}{q} \pi\right)=\cos \left((2 j-1) \pi+\frac{p}{q} \pi\right)=\cos \left(\frac{p}{q} \pi\right) .
$$

Such a $j$ always exists since the difference of its upper and lower limits is

$$
\frac{2 q-p}{2(q-p)}-\frac{p}{2(q-p)}=1
$$

and neither of them is an integer since the nominators are odd and the denominators are even.

(ii) If $q<p<2 q$ then choose $j$ according to

$$
\frac{2 q-p}{2(q-p)}<j<\frac{p}{2(q-p)}
$$

The same algebraic and trigonometric manipulation as above leads to the same result (A.2).

(iii) If $2 n q<p<(2 n+1) q$ for some $n=1,2, \ldots$ then define

$$
\tilde{p}=p-2 n q \quad \Rightarrow \cos \left(\frac{\tilde{p}}{q} \pi\right)=\cos \left(\frac{p}{q} \pi\right)
$$

Now, $j$ can be chosen with the help of $\tilde{p}$ in the same way as in case $i$ :

$$
\frac{\tilde{p}}{2(q-\tilde{p})}<j<\frac{2 q-\tilde{p}}{2(q-\tilde{p})}
$$

which leads to (A.2), again.

(iv) Finally, if $(2 n+1) q<p<(2 n+2) q$ for some $n=1,2, \ldots$ then define $\tilde{p}$ and choose $j$ in the following way:

$$
\tilde{p}=p-(2 n+1) q, \quad \frac{2 q-\tilde{p}}{2(q-\tilde{p})}<j<\frac{\tilde{p}}{2(q-\tilde{p})} .
$$

With this $j,(\mathrm{~A} .2)$ is true again.

This way, for all the possible combinations of the coprimes $p, q$ where $p$ is odd and $q \neq 1$, we proved that there exists $j$ such that $S(j-1) S(j)=-1$, that is, the system is unstable. Clearly, for odd $p$ and $q=1, S(j-1) S(j)=+1$ in $(A .1)$ for any $j$, which is in accordance with the identified stability regions in Section 5.3.1.

\section{References}

[1] G. Domokos, P. Holmes, Euler's problem, Euler's method, and the standard map; or, the discrete charm of buckling, Journal of Nonlinear Science 3 (1993) 109-151.

[2] P. Holmes, G. Domokos, J. Schmitt, I. Szeberenyi, Constrained Euler buckling: an interplay of computation and analysis, Computer Methods in Applied Mechanics and Engineering 170 (1999) 175-207.

[3] K. Ito, J. Turi, Numerical methods for a class of singular integro-differential equations based on semigroup approximation, SIAM Journal on Numerical Analysis 28 (1991) 1698-1722.

[4] K. Ito, F. Kappel, The Trotter-Kato theorem and approximation of PDEs, Mathematics of Computation 67 (1998) 21-44.

[5] M. Haraguchi, H.Y. Hu, Stability analysis of a noise control system in a duct by using delay differential equation, Acta Mechanica Sinica 25 (2009) $131-137$.

[6] T. Schmitz, The microphone feedback analogy for chatter in machining, Shock and Vibration 2015 (2015) 1-5, Article ID 976819.

[7] H. Ma, E.A. Butcher, Stability of elastic columns with periodic retarded follower forces, Journal of Sound and Vibration 286 (2005) 849-867.

[8] M. Haraguchi, H.Y. Hu, Vibration suppression of flexible beam with delayed boundary feedback via discrete-time optimal controller, IEEE International Conference on Mechatronics and Automation, Conference Proceedings, 2007, pp. 158-162.

[9] M. Kidd, G. Stepan, Delayed control of an elastic beam, International Journal of Dynamics and Control 2 (2014) 68-76.

[10] M. Fliesst, H. Mouniert, P. Rouchon, J. Rudolph, Controllability and motion planning for linear delay systems with an application to a flexible rod, Proceedings of the IEEE 34th Conference on Decision \& Control, 1995, pp. 2046-2051.

[11] R. Szalai, Impact mechanics of elastic structures with point contact, ASME Journal of Vibration and Acoustics 136 (2014) 1715-1730.

[12] T. Takemura, T. Kitamura, T. Hoshi, K. Okushima, Active suppression of chatter by programmed variation of spindle speed, Annals of the CIRP 23 (1974) $121-122$.

[13] T. Insperger, G. Stepan, Stability analysis of turning with periodic spindle speed modulation via semi-discretisation, Journal of Vibration and Control 10 (2004) 1835-1855.

[14] W.M. Leach, Introduction to Electroacoustics and Audio Amplifier Design, Kendall/Hunt Publishing Co, Dubuque, 2003. 
[15] J. Benesty, T. Gänsler, D. Morgan, M. Sondhi, S. Gay, Advances in Network and Acoustic Echo Cancellation, Springer, Berlin, 2001.

[16] A. Mader, H. Puder, G.U. Schmidt, Step-size control for acoustic echo cancellation filters-an overview, Signal Processing 80 (2000) 1697-1719.

[17] P.A. Nelson, S.J. Elliott, Active Control of Sound, Academic Press, New York, 1991.

[18] C.R. Fuller, A.H. von Flotow, Active control of sound and vibration, IEEE Control Systems Magazine 15 (1995) 9-19.

[19] S.M. Kuo, D.R. Morgan, Active noise control: a tutorial review, Proceedings of the IEEE 87 (1999) 943-973.

[20] M.J. Brennan, K.A. Ananthaganeshan, S.J. Elliott, Instabilities due to instrumentation phase-lead and phase-lag in the feedback control of a simple vibrating system, Journal of Sound and Vibration 304 (2007) 466-478.

[21] Y. Sun, J. Xu, Experiments and analysis for a controlled mechanical absorber considering delay effect, Journal of Sound and Vibration 339 (2015) $25-37$.

[22] R. Datko, J. Lagnese, M. Polis, An example on the effect of time delays in boundary feedback stabilization of wave equations, SIAM Journal on Control and Optimization 24 (1986) 152-156.

[23] R. Datko, Not all feedback stabilized hyperbolic systems are robust with respect to small time delays in their feedbacks, SIAM Journal on Control and Optimization 26 (1988) 697-713.

[24] Y.N. Kyrychko, S.J. Hogan, On the use of delay equations in engineering applications, Journal of Vibration and Control 16 (2010) 943-960.

[25] W. Michiels, S. Niculescu, Stability and Stabilization of Time-Delay Systems: An Eigenvalue-based Approach, SIAM, Philadelphia, 2007.

[26] N. Olgac, R. Sipahi, The cluster treatment of characteristic roots and the neutral type time-delayed systems, ASME Journal of Dynamic Systems, Measurement and Control 127 (2005) 88-97.

[27] R. Sipahi, N. Olgac, Complete stability analysis of neutral-type first order two-time-delay systems with cross-talking delays, SIAM Journal of Control and Optimization 45 (2006) 957-971.

[28] J.K. Hale, A.M. Verduyn Lunel, Strong stabilization of neutral functional differential equations, IMA Journal of Mathematical Control and Information 19 (2002) 5-23.

[29] J.K. Hale, Theory of Functional Differential Equations, Springer-Verlag, New York, 1977.

[30] G. Stepan, Retarded Dynamical Systems, Longman Group, New York, 1989.

[31] W.J. Stronge, Impact Mechanics, Cambridge University Press, Cambridge, 2000.

[32] G. Stepan, Z. Szabo, Impact induced internal fatigue cracks, Proceedings of the ASME Design Engineering Technical Conferences, 17th Biennial Conference on Mechanical Vibration and Noise, Las Vegas, September 12-15, 1999, pp. 1-7, Article no. DETC99/VIB-8351.

[33] C.W. de Silva, Vibrations: Fundamentals and Practice, Taylor and Francis, Boca Raton, 2007.

[34] D. Kiusalaas, D. Davis, On the stability of elastic systems under retarded follower forces, International Journal of Solids and Structures 6 (1970) 399-409.

[35] L. Perneder, E. Detournay, Steady-state solutions of a propagating borehole: helical trajectory, 20th IEEE International Conference on Control Applications (CCA), Denver, September 28-30, 2011, pp. 905-911.

[36] G.C. Downton, Directional drilling system response and stability, 16th IEEE International Conference on Control Applications (CCA), Singapore, October 13, 2007, pp. 1543-1550.

[37] J. Munoa, I. Mancisidor, N. Loix, L.G. Uriarte, R. Barcena, M. Zatarain, Chatter suppression in ram type travelling column milling machines using a biaxial inertial actuator, CIRP Annals - Manufacturing Technology 62 (2013) 407-410.

[38] D. Lehotzky, J. Turi, T. Insperger, Stabilizability diagram for turning processes subjected to digital PD control, International Journal of Dynamics and Control 2 (2014) 46-54. 